\title{
Grid Computing Technologies for Renewable Electricity Generator Monitoring and Control
}

\author{
P R Hobson, E Frizziero, Chenxi Huang, M R Irving, T Kalganova, P Kyberd, , F Lelli, A Petrucci, \\ R Pugliese, G Taylor, R Taylor
}

\begin{abstract}
In this paper we discuss the use of real-time Grid computing for the monitoring, control and simulation of renewable electricity generators and their associated electrical networks. We discuss briefly the architectural design of GRIDCC and how we have integrated a number of real (solar, CHP) and simulated conventional power generators into the GRIDCC environment. A local weather station has also been attached to an Instrument Manager to alert experts appropriately when the Solar Array is not generating. The customised remote control and monitoring environment (a virtual control room), distributed using a standard web server, is discussed.
\end{abstract}

Index Terms - Distributed Computing, Electrical Power Generation, Grid Computing, Renewable Energy

\section{INTRODUCTION}

Grid computing has recently been developed primarily to solve large-scale computational challenges in areas such as genomics, biomedicine, particle physics and other scientific fields with similar large-scale computational challenges. The EU funded GRIDCC project [1] is extending the concept of the Grid to encompass virtualized instrument resources and to create middleware which can provide Grids with a real-time quality of service.

There are many application areas driving the development of a Grid that includes resources beyond the compute and storage elements of currently deployed Grids. One prototype application being developed in the GRIDCC project is control and monitoring of renewable electricity generation.

In the coming decades a large number of offshore wind power generators will be connected to European transmission systems [2]. As these generators contribute larger amounts of power it will become necessary to monitor their performance and control their operation with a much higher degree of accuracy, reliability and cooperation between transmission

Manuscript received March 30, 2007. This work was supported in part by the EC Framework Programme 6 Contract IST-511382.

P R Hobson, Chenxi Huang, M R Irving, T Kalganova, P Kyberd, G Taylor, R Taylor are with the School of Engineering \& Design, Brunel University, Uxbridge UB8 3PH, UK (corresponding author P R Hobson: $+44(0) 1895266799$; fax: $\quad+44(0) 1895272391$; $\quad$ e-mail: Peter.Hobson@brunel.ac.uk).

E Frizziero, F Lelli and A Petrucci. are with INFN, Via Romea, 4, Legnaro, I-35020, Italy

R Pugliese is with Sincrotrone Trieste S.C.p.A. di interesse nazionale, Strada Statale 14 km 163 in AREA Science Park, Basovizza, Trieste I-34012, Italy

1-4244-0635-8/07/\$20.00@2007 IEEE system operators. The standard control technologies that are currently in use at transmission level are not easily scalable to the very large numbers of off-shore wind power generators which will be operating across Europe in the future. Open Source Grid computing can provide a relatively inexpensive solution, allowing the output of wind power generators to be monitored and when appropriate controlled across large geographical areas. An outline of the power systems Gridcomputing platform that has been adopted for our research is illustrated in Figure 1.

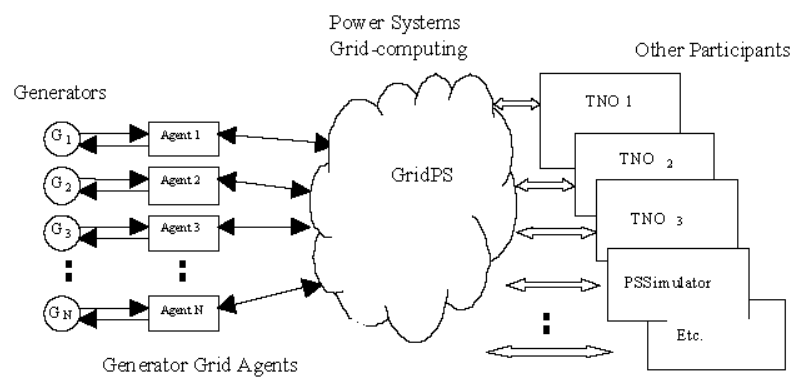

Fig. 1. Overview of a power systems Grid Computing platform (from [3]).

A Grid is scaleable - inherently it is designed to work as well with 100,000 participants (nodes) as it is with 100 . The distribution of computing resources requires additional network security, and it is important to note that this is also inherent to Grid computing, yet it can still provide a robust provision of computational resources in terms of selforganisation and major fault tolerance [4]. Data can also be distributed and replicated across a network of a virtual organisation with specified levels of security.

\section{GRIDCC}

The GRIDCC project has now demonstrated the extension of existing computational grids to provide the ability to control and monitor collections of real instruments with accompanying supporting services such as a virtual control room (VCR) a workflow management system (WfMS), and a generic Problem Solver running locally or globally. Some care has been taken to build into the GRIDCC concept the requirements of real-time systems, where here real-time implies a defined response time for a process to start/complete/return a status message etc. rather than any particular data rate. A critical element in achieving real-time 
response is the quality of service repository which can be queried both by the Execution Service and from the VCR.

Figure 2 shows the architecture of GRIDCC and figure 3 shows the internal details of its new contribution to Grid computing; the instrument element (IE) [5].

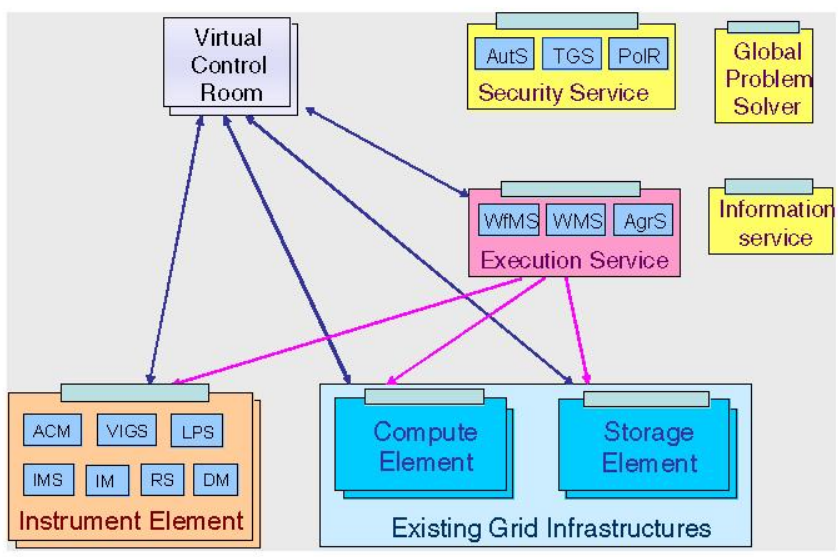

Fig. 2. Architecture of GRIDCC. The Execution Service controls the Instrument Element and existing Grid resources using a workflow management system (WfMS) and agreement service (AgrS). The Instrument Element is connected by instances of the Instrument Manager (IM) to real sensors and instruments.

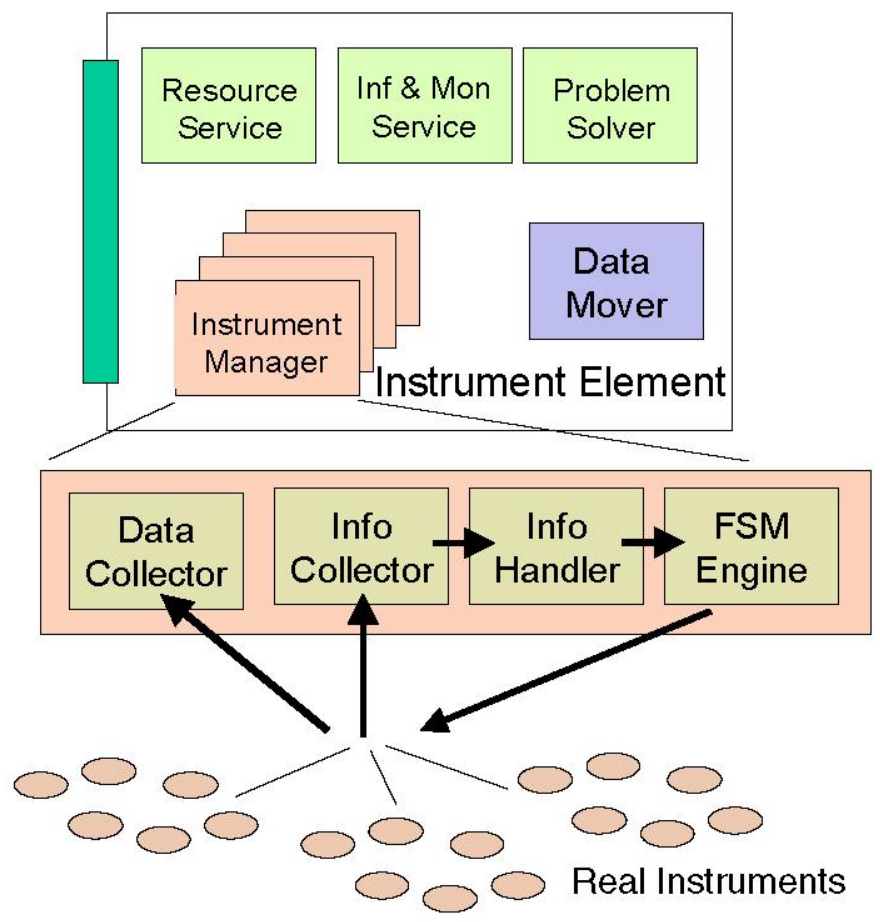

Fig. 3. The internal details of the Instrument Element. The Instrument Manager handles the control, command and data for a specific instrument.

The IE is a coherent collection of services allowing remote configuration and control of a physical instrument and integration with existing computational Grids. Inside the IE a set of Web Service interfaces called VIGS (Virtual Instrument Grid Service) allows the user to remotely access the real instrument, thus plugging the instrument itself in to the Grid.

Additionally the Instrument Element can provide facilities for interactive co-operation between computing Grid resources to applications that have real-time requirements or need fast interaction with Compute Elements (CE) and Storage Elements (SE).

The Multipurpose Collaborative Environment (MCE) is Web-based groupware which provides collaboration support tools and the foundation to allow teams of people to control and manage Grid resources, including remote instrumentation. The MCE has been designed taking into account the fundamental needs of end-users and stakeholders of pilot applications. In particular, a modular and layered architecture supports the common requirements of a range of pilot applictations. Following this philosophy, the MCE design and development has been divided into three parts:

- a core system, providing fundamental architecture and services of the entire system;

- a set of general purpose plug-ins, aimed at supporting common groupware functionalities (e.g. chat, shared calendars, integration with videoconferencing systems), that can be activated/deactivated (or even substituted with other ones) in each specific VCR;

- a set of application-specific plug-ins, supporting functionalities that are needed only for particular applications (e.g. the control of a particular instrument).

The MCE is a Web portal providing access to the typical functionalities of a scientific groupware through a Webbrowser. Among the suitable portal frameworks available, GridSphere [6] offered a complete set of features (e.g. portal and user administration functionalities, JSR 168 standard compliance, support for the integration of Grid components) for the goals of the GRIDCC project.

\section{DEMONSTRATOR For ELECTRICAL GENERATOR MONITORING \& CONTROL}

At Brunel University there exist a number of small renewable energy generators, currently two $500 \mathrm{~W}$ photovoltaic (PV) arrays and a $150 \mathrm{~kW}$ combined heat \& power (CHP) of which $80 \mathrm{~kW}$ is electrical. A small (few $\mathrm{kW}$ ) wind turbine is envisaged and we have a local weather station for environmental monitoring. In addition to the real generators we have sophisticated computer models [7] of electrical generators which we can run on Grid cluster worker nodes in order to evaluate the scalability of the GRIDCC control and monitoring system to potentially thousands of generator instances.

Currently we have customized Instrument Managers for the three classes of power generator and the weather station. Each of the generators has a different interface to the Instrument Element via individual Instrument Managers. The generators have finite state machines (FSM Engine in Figure 3) to enable them to be control from the VCR (see figure 4) or the WfMS. 


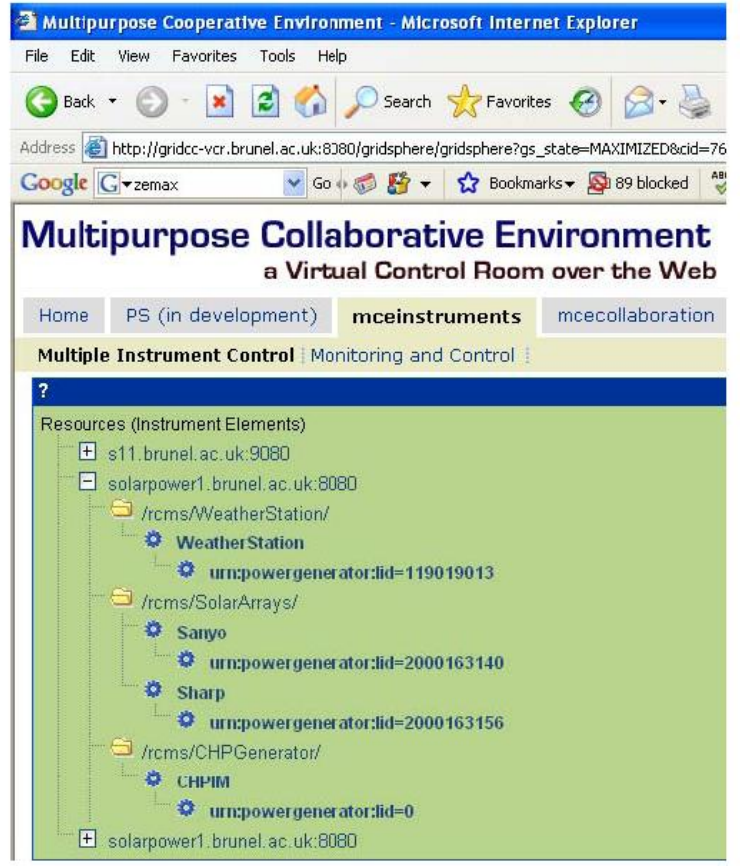

Fig. 4. The Instrument view presented to a remote user by the MCE. Here you can see the links to the Weather Station, the two PV arrays (Sanyo and Sharp) and the CHP Generator.

Figure 5 shows the monitoring of the electrical power output of one of the PV arrays on a sunny day. The data is updated every 5 seconds.

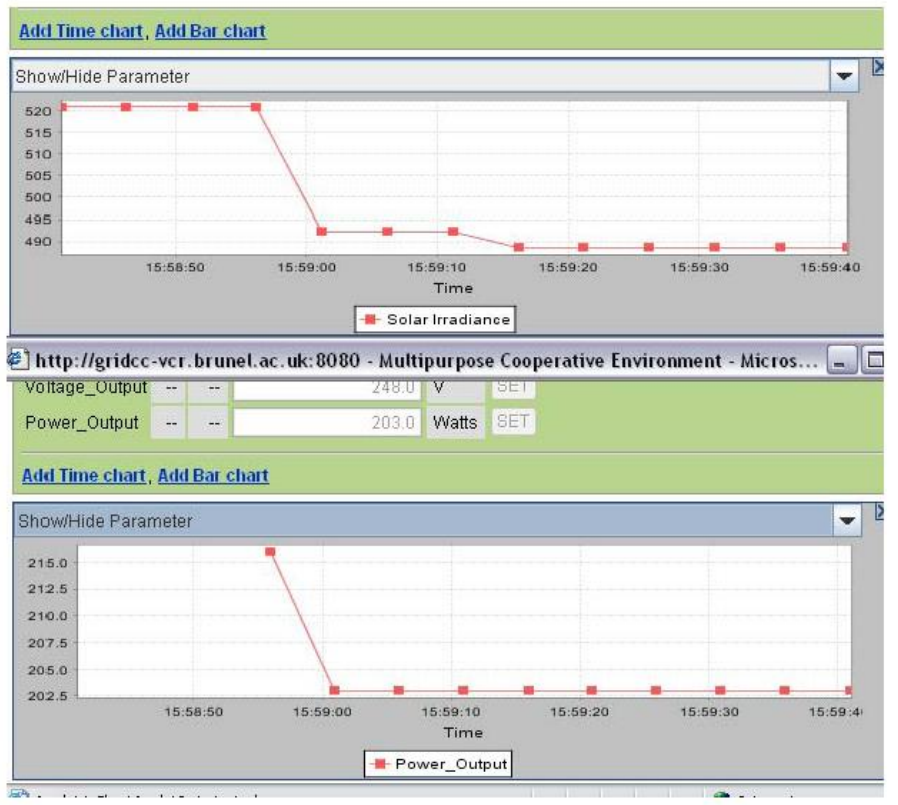

Fig. 5. The monitored data from one of the PV arrays. The Solar Irradiance $\left(\mathrm{W} \cdot \mathrm{m}^{-2}\right)$ is shown on the upper time chart and the simultaneously measured instantaneous electrical power in watts is shown on the lower trace. The correlated drop at 15:58:56 UT+1 is clearly seen.

The ability of a remote operator (or multiple remote experts) to see the dynamic behavior of any of the generators at any time is a principle goal of this project. A problem solver framework that can operate at the level of the Instrument Element (see figure 3 ) is currently being implemented. This allows any appropriate problem solving system, for example a neural network or a decision tree, to be used to determine automatically what the likely problem is when a generator ceases to behave normally (for example sudden loss of power). Local remedial action, for example a shutdown and restart, could be taken or an appropriate message sent to remote experts. The collaborative features of the MCE, such as the use of video conferencing facilities (provided by the VRVS tool $[8,9]$ ) or email, chat window or Skype [10] telephone call also enhance the interaction between the control centre and the remote sites.

\section{Conclusion}

This paper reports on work in progress with a pilot application using the GRIDCC middleware for the remote control and monitoring of both real and simulated electrical power generators. We have reported on the deployment of key elements of the GRIDCC middleware, namely the Instrument Element which virtualizes real instruments and sensors into a Grid computing resource analogous to a computational Worker Node and the Multipurpose Collaborative Environment which provides a customizable web portal based on GridSphere from which individual instruments can be monitored and controlled.

We are now deploying other parts of the GRIDCC middleware, namely the Work-Flow Management System and the Local Problem Solver to deal with use cases such as a Power Network Operator requesting an increase in power generation due to increased load demand. Providing a realtime quality-of-service is an important final stage of implementation in the demonstrator.

We envisage the application to renewable electricity generator monitoring and control to be aimed primarily at small to medium scale systems where existing dedicated network links and bespoke commercial software systems may be too expensive compared to the use of the GRIDCC open source middleware. Any existing local control system can be wrapped up by an Instrument Manager so that it can be operated remotely by the a GRIDCC-based system. This is precisely what we have done in the demonstrator described above with the commercial control and monitor systems that were provided for the Solar Arrays and the CHP.

\section{ACKNOWLEDGMENT}

This abstract is submitted on behalf of the whole GRIDCC collaboration.

\section{REFERENCES}

[1] GRIDCC EU ICT Project, 'Grid Enabled Remote Instrumentation with Distributed Control and Computation. Available http://www.gridcc.org/

[2] R. Pool, "Meet the New Super Power," IET Power Engineer, pp. 8-9, June/July. 2006.

[3] G.A. Taylor et al, "Scalable Integration of Wind Power on Transmission Systems u sing Grid Computing," in Sixth International Workshop on Large-Scale Integration of Wind Power and Transmission Networks for Offshore Wind Farms, Delft, 2006, pp. 26-28.

[4] Foster, C. Kesselman and S. Tuecke, "The Anatomy of the Grid: Enabling Scalable Virtual Organisations" Int. Jnl. of High Performance Computing Applications. vol. 15, 2001, pp. 200-222 
[5] E Frizziero et al, "Instrument Element: A New Grid component that Enables the Control of Remote Instrumentation," in Sixth IEEE International Symposium on Cluster Computing and the Grid Workshops (CCGRIDW'06), SIngapore, 2006, DOI Bookmark: http://doi.ieeecomputersociety.org/10.1109/CCGRID.2006.146

[6] GridSphere Portal Framework Available http://www.gridsphere.org/gridsphere/gridsphere

[7] M.Rafian, M.J.H.Sterling and M.R.Irving, "Real Time Power System Simulation," Proc. IEE C 134, pp 206-223, 1987

[8] D. Adamczyk, et al, "A Globally Distributed Real Time Infrastructure for World Wide Collaborations," in Proceedings of Computing for High Energy Physics (CHEP), Interlaken, 2004 Available http://indico.cern.ch/getFile.py/access? contribId=88\&amp; sessionId=11 \&amp;resId $=0$ \&amp; materialI $\$=$ paper \&amp; $\operatorname{confI}=0$

[9] Virtual Room Videoconferencing System Available http://www.vrvs.org/

[10] skype Available http://www.skype.com/intl/en-gb/ 